ARTICLE

\title{
A bibliometric study of research topics, collaboration, and centrality in the iterated prisoner's dilemma
}

\author{
Nikoleta E. Glynatsi ${ }^{1,2}{ }^{凶}$ \& Vincent A. Knight ${ }^{1}$
}

This manuscript explores the research topics and collaborative behaviour of authors in the field of the Prisoner's Dilemma using topic modeling and a graph theoretic analysis of the coauthorship network. The analysis identified five research topics in the Prisoner's Dilemma which have been relevant over the course of time. These are human subject research, biological studies, strategies, evolutionary dynamics on networks and modeling problems as a Prisoner's Dilemma game. Moreover, the results demonstrated the Prisoner's Dilemma is a field of continued interest, and that it is a collaborative field compared to other game theoretic fields. The co-authorship network suggests that authors are focused on their communities and that not many connections across the communities are made. The most central authors of the network are the authors connected to the main cluster. Through examining the networks of topics, it was uncovered that the main cluster is characterised by the collaboration of authors in a single topic. These findings add to the bibliometrics study in another field and present new questions and avenues of research to understand the reasons for the measured behaviours.

\footnotetext{
${ }^{1}$ School of Mathematics, Cardiff University, Cardiff, United Kingdom. ${ }^{2}$ Max Planck Institute for Evolutionary Biology, Plön, Germany.

凶email: glynatsi@evolbio.mpg.de
} 


\section{Introduction}

he Prisoner's Dilemma (PD) is a well known game used since its introduction in the 1950's (Flood, 1958) as a framework for studying the emergence of cooperation; a topic of continued interest for mathematical, social, biological and ecological sciences. This manuscript presents a bibliometric analysis of 2420 published articles on the Prisoner's Dilemma between 1951 and 2018. It presents a number of research topics in the PD publications, which have been identified using Latent Dirichlet Allocation (LDA) (Blei et al., 2003), and it explores the changes in the research topics over time. The collaborative behaviour of the field is explored using the co-authorship network, and furthermore, the LDA topic analysis is combined with the co-authorship network analysis to assess the most central authors in these topics. Assessing the collaborative behaviour of the field of collaboration itself is the main aim of this work.

As discussed in (Youngblood and Lahti, 2018), bibliometrics (the statistical analysis of published works originally described by (Pritchard et al., 1969)) has been used to support historical assumptions about the development of fields (Raina and Gupta, 1998), identify connections between scientific growth and policy changes (das Neves Machado et al., 2016), develop a quantitative understanding of author order (Sekara et al., 2018), and investigate the collaborative structure of an interdisciplinary field (Liu and Xia, 2015). Most academic research is undertaken in the form of collaborative effort and as (Kyvik and Reymert, 2017) points out, it is rational that two or more people have the potential to do better as a group than individually. Indeed this is the very premise of the PD itself. Collaboration in groups has a long tradition in experimental sciences and it has be proven to be productive according to (Etzkowitz, 1992). The number of collaborations can be different between research fields and understanding how collaborative a field is not always an easy task. Several studies tend to consider academic citations as a measure for these things. A blog post published by Nature (van Noorden, 2017) argues that depending on citations can often be misleading because the true number of citations can not be known. Citations can be missed due to data entry errors, academics are influenced by many more papers than they actually cite and several of the citations are superficial.

A more recent approach to measuring collaborative behaviour, and to studying the development of a field is to use the coauthorship network, as described in (Liu and Xia, 2015). The co-authorship network has many advantages as several graph theoretic measures can be used as proxies to explain author relationships. For example the average degree of a node corresponds to the average number of an authors' collaborators, and clustering coefficient corresponds to the extent that two collaborators of an author also collaborate with each other. In (Liu and Xia, 2015), the approach was applied to analyse the development of the field "evolution of cooperation", and in (Youngblood and Lahti, 2018) to identify the subdisciplines of the interdisciplinary field of "cultural evolution" and investigate trends in collaboration and productivity between these subdisciplines. Moreover, (Li et al., 2019) examined the long-term impact of co-authorship with established, highly-cited scientists on the careers of junior researchers.

LDA is a topic modeling technique proposed in (Blei et al., 2003) as a generative probabilistic model for discovering underlying topics in collections of data. Applications of the technique include detection in image data (Coelho et al., 2010) and detection in video (Wang et al., 2008). Nevertheless, LDA has been applied by several works on publication data for identifying the topic structure of a subject area. In (Inglis and Foster, 2018), it was applied to the publications on mathematical education of the journals "Educational Studies in Mathematics" and "Journal for
Research in Mathematics Education" to identify the dominant topics that each journal was publishing on. The topics of the North American library and Information Science dissertations were studied chronologically in (Sugimoto et al., 2011), and the main topic of the scientific content presented at EvoLang conferences was identified in (Bergmann and Dale, 2018). In (Bergmann and Dale, 2018) the LDA approach is combined with clustering and a co-authorship network analysis. A clustering analysis is applied to the LDA topics, and the co-authorship network is analysed as a whole where the clusters are only used to differentiate between the authors' topics.

This paper builds upon the previous works of (Bergmann and Dale, 2018, Liu and Xia, 2015, Youngblood and Lahti, 2018). It extends their methodology, it combines identified topics by an LDA model with the co-authorship network analysis, and applies all these techniques to a new data set. This data set was collected not from a single source but from five different sources. The four publishers were chosen because they are well known publishers in the field, and the arXiv preprint server. The search terms used to collect data appear on relevant articles and the search fields that were used were the title, abstract and text. However, papers can refer to the PD in the text but not analyze the topic. For this reason such articles were manually checked, so that only relevant papers are included in the analysis. Moreover, an amount of well known articles, which are not published in any of the selected publishers, were manually included in the data set.

The methodology used in this manuscript, which includes the data collection and a preliminary analysis of the data set, is covered in Section "Methodology". The results on the research topics of the PD are presented in Section "Research topics in the Prisoner's Dilemma research", and the results on the coauthorship network are presented in Section "Analysis of coauthorship network". Finally, the conclusions are summarised in Section "Conclusion".

\section{Methodology}

Academic articles are accessible through scholarly databases. Several databases and collections today offer access through an open application protocol interface (API). An API allows users to query directly a publisher's database and bypass the graphical user interface. Interacting with an API has two phases: requesting and receiving. The request phase includes composing a url with the details of the request. For example, http://export.arxiv.org/ api/query?search_query=abs:prisoner'sdilemma\&max_results $=1$ represents a request message. The first part of the request is the address of the API. In this example the address corresponds to the API of arXiv. The second part of the request contains the search arguments. In this example it is requested that the word 'prisoners dilemma' exists within the article's title. The format of the request message is different from API to API. The receive phase includes receiving a number of raw metadata of articles that satisfies the request message. The raw metadata are commonly received in extensive markup language (xml) or Javascript object notation (json) formats (Nurseitov et al., 2009). Similarly to the request message, the structure of the received data differs from publisher to publisher.

The data collection is crucial to this study. To ensure that this study can be reproduced all code used to query the different publishers' APIs has been packaged as a Python library and is available online (Glynatsi, 2017). The software could be used for any type of projects similar to the one described here, documentation for it is available at: http://arcas.readthedocs.io/ en/latest/. Project (Glynatsi, 2017) can collect data from five 
different sources. These correspond to four publishers and a preprint server:

- $\quad \operatorname{arXiv}$ (McKiernan, 2000); a repository of electronic preprints. It consists of scientific papers in the fields of mathematics, physics, astronomy, electrical engineering, computer science, quantitative biology, statistics, and quantitative finance, which all can be accessed online.

- PLOS (PLOS, 2000); a library of open access journals and other scientific literature under an open content license. It launched its first journal, PLOS Biology, in October 2003 and publishes seven journals, as of October 2015.

- IEEE Xplore Digital Library (IEEE) (IEEE, 1963); a research database for discovery and access to journal articles, conference proceedings, technical standards, and related materials on computer science, electrical engineering and electronics, and allied fields. It contains material published mainly by the Institute of Electrical and Electronics Engineers and other partner publishers.

- Nature (Nature Publishing Group, 1869); a multidisciplinary scientific journal, first published on 4 November 1869. It was ranked the world's most cited scientific journal by the Science Edition of the 2010 Journal Citation Reports and is ascribed an impact factor of 40.137, making it one of the world's top academic journals.

- Springer (Mannheim Media, 1950); a leading global scientific publisher of books and journals. It publishes close to 500 academic and professional society journals.

These publishers were chosen because they are prominent publishers in the field. For each source, data can be collected by specifying a search term and a search field. Articles for which any of the terms:

- prisoner's dilemma

- prisoners dilemma

- prisoner dilemma

- prisoners evolution

- prisoner game theory

existed within the title, the abstract or the text are included in the analysis. These terms we selected because they are occurring terms in paper known to be relevant in the field. However, the authors acknowledge that there are other terms that could have been used, for example "donation game". The authors believe that the results of the manuscript do generalise to the overall stated goals (Section "Introduction"), but they are inferred only from the data collected on the specific search terms and search fields.

The latest data collection was performed on the 30th November 2018. Following the automatic collection of articles from the sources, a cleaning process was applied to the data. More specifically, all the titles of the collected articles were compared for semantic similarity. There were a total of 34 duplicate articles. That was because both the preprint and the published versions of a paper were collected. The preprint versions (collected from arXiv) were dropped at this stage. A semantic similarity check was also applied in the names of the collected authors. The names that were highlighted as similar were manually checked. In case of a duplicate, for example "Martin Nowak" and "Martin A. Nowak" are considered duplicates, all entries of that author were fixed to a single style. Most commonly the middle name was dropped. Finally, articles that were collected because the search terms existed within the text were checked to reassure their relevance to the PD topic. Non relevant articles were dropped at this stage.

Following the cleaning process, a total of 76 articles were manually added to the data set because they are of interest to the field. This was also done in (Liu and Xia, 2015). Examples of such papers include (Flood, 1958) the first publication on the PD, (Ohtsuki et al., 2006, Stewart and Plotkin, 2012) two well cited articles in the field, and a series of works from Robert Axelrod (Axelrod, 1980a),(Axelrod, 1980b),(Axelrod et al., 1987),(Axelrod and Hamilton, 1981),(Riolo et al., 2001) a leading author of the field. The process of obtaining the data set used in analysis presented in the manuscript is illustrated in Fig. 1. This data set has been archived and is available at (Glynatsi, 2019c).

The data set consists of 2422 articles with unique titles. A more detailed summary of the articles' provenance is given by Table 1 . Only $3 \%$ of the data set consists of articles that were manually added and $27 \%$ of the articles were collected from arXiv. The average number of publications is also included in Table 1. Overall an average of 43 articles are published per year on the topic. The most significant contribution to this appears to be from arXiv with 11 articles per year, followed by Springer with 9 and PLOS with 8 .

All the visualisations presented in the manuscript were generated using (Hunter, 2007), and project (Walt et al., 2011) was used for manipulating the data.

The data handled here is in fact a time series from the 1950s, the formulation of the game, until 2018 (Fig. 2). Two observations can be made from Fig. 2 .

1. There is a steady increase of the number of publications since the 1980 s and the introduction of computer tournaments (Axelrod and Hamilton, 1981) (work by Robert Axelrod).

2. There is a decrease in 2017-2018. This is due to our data set being incomplete. Articles that have been written in 2017-2018 have either not being published or were not retrievable by the APIs at the time of the last data collection.

These observations can be confirmed by studying the time series. Using (Jones et al., 2001), an exponential distribution is fitted to the data. The fitted model can be used to forecast the behaviour of the field for the next 5 years. Even though the time series has indicated a slight decrease, the model forecasts that the number of publications will keep increasing, thus demonstrating that the field of the PD continues to attract academic attention.

There are a total of 4226 authors in the data set (Glynatsi, 2019c) and several of these authors have had multiple publications collected from the data collection process. The highest number of articles collected for an author is 83 publications for Matjaz Perc. However, Matjaz Perc is an outlier most authors have 1 to 6 publications in the data set. The overall Collaboration Index (CI) or the average number of authors on multi-authored papers is 3.2, thus on average a non single author publication in the PD has 3 authors. This appears to be quite standard compared to other fields such as cultural evolution (Youngblood and Lahti, 2018), Astronomy and Astrophysics, Genetics and Heredity, Nuclear and Particle Physics as reported by (Mallapaty, 2018). There are only a total of 545 publications with a single author, which corresponds to the $22 \%$ of the papers. It appears that academic publications tend to be undertaken in the form of collaborative effort, which is in line with the claim of (Kyvik and Reymert, 2017).

The collaborativeness of the authors is explored in more detail in Section "Analysis of co-authorship network" using the coauthorship network. The collaborative behaviour of authors will also be explored at the research topics level. These topics and their relevance over time are presented in Section "Research topics in the Prisoner's Dilemma research".

\section{Results}

Research topics in the prisoner's dilemma research. The articles contained in the data set (Glynatsi, 2019c) are classified into research topics using $\mathrm{LDA}$, an unsupervised machine learning 


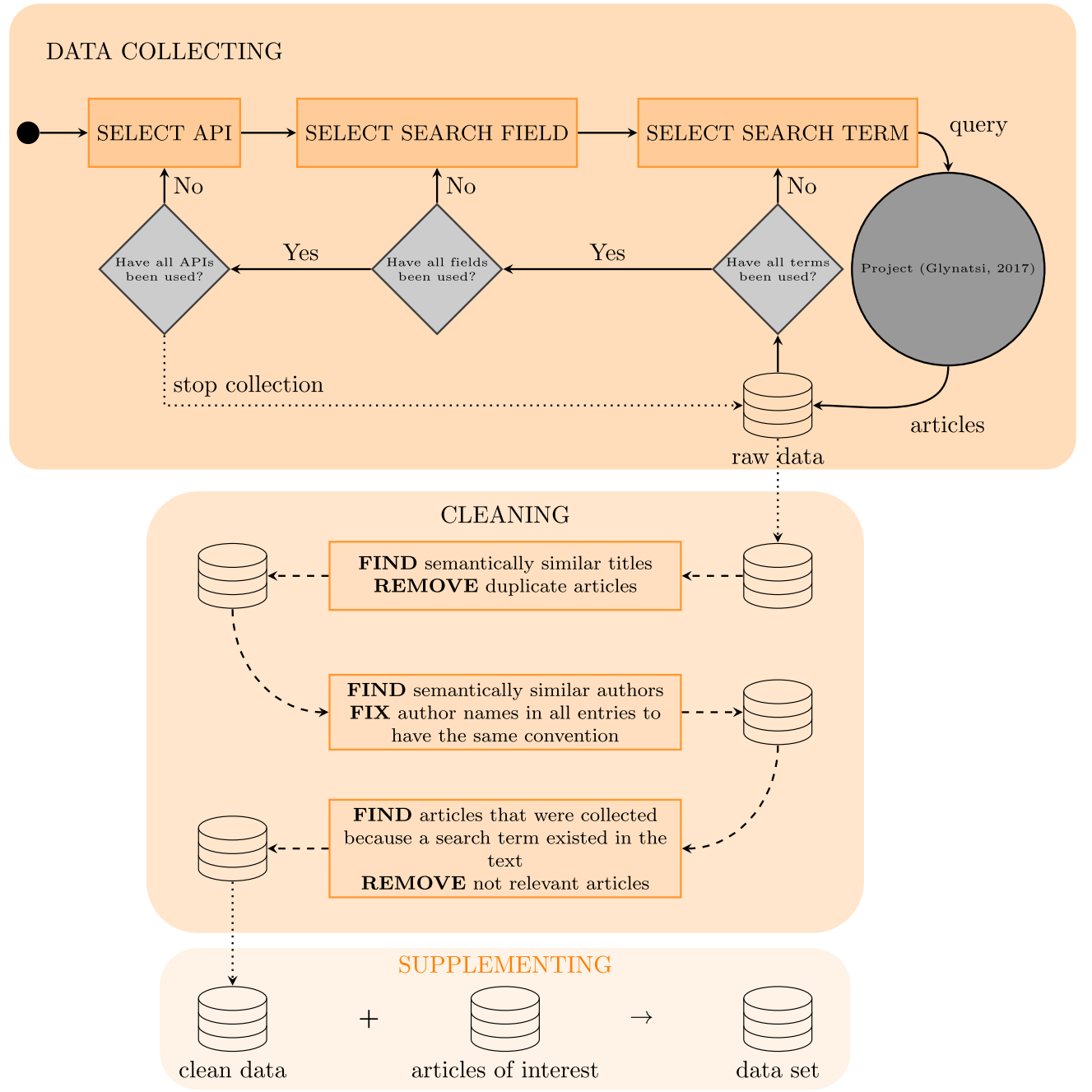

Fig. 1 The generating process of the data set (Glynatsi, 2019c). Diagrammatic representation of the data generating process.

\begin{tabular}{|lllll|}
\hline \multicolumn{2}{|c|}{ Table 1 Summary of (Glynatsi, 2019c) per provenance. } \\
\hline & $\begin{array}{l}\text { Number } \\
\text { of articles }\end{array}$ & Percentage \% & $\begin{array}{l}\text { Year of first } \\
\text { publication }\end{array}$ & $\begin{array}{l}\text { Average } \\
\text { number of } \\
\text { publications } \\
\text { per year }\end{array}$ \\
\hline IEEE & 294 & $12.14 \%$ & 1973 & 5 \\
Manual & 76 & $3.14 \%$ & 1951 & 1 \\
Nature & 436 & $18.00 \%$ & 1959 & 8 \\
PLOS & 477 & $19.69 \%$ & 2005 & 8 \\
Springer & 533 & $22.01 \%$ & 1966 & 9 \\
arXiv & 654 & $27.00 \%$ & 1993 & 11 \\
Overall & 2470 & $100.00 \%$ & 1951 & 43 \\
\hline
\end{tabular}

technique designed to summarize large collections of documents by a small number of conceptually connected topics or themes (Blei et al., 2003, Grimmer and Stewart, 2013). The documents are the articles' abstracts and LDA was carried out using (Rehurek and Sojka, 2010). In LDA, each document/ abstract is represented by a distribution over topics, and the topics themselves are represented by a distribution over words. More specifically, each topic is described by weights associated with words and each document by the probabilities of belonging to a specific topic. The probability of a document belonging to topic $T$ is referred to as the percentage

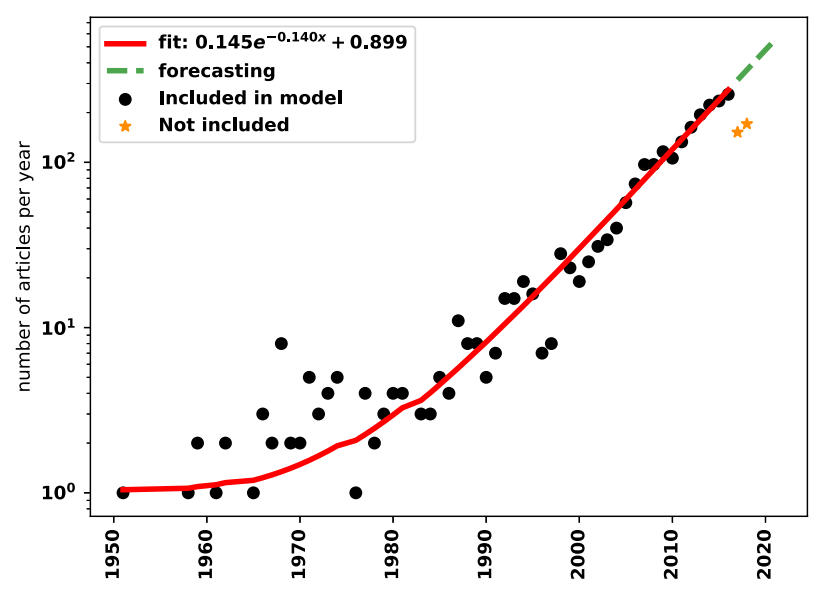

Fig. 2 Number of published articles on the PD over time. The number of articles published on the PD 1951-2018 on a log scale, with a fitted exponential line, and a forecast for 2017-2022.

contribution denoted as $c_{T}$. For example the words and their associated weights for two topics A and B could be:

- Topic A: $0.039 \times$ “cooperation", $0.028 \times$ "study", and $0.026 \times$ "human". 
- Topic B: $0.020 \times$ “cooperation", $0.028 \times$ “agents", and $0.026 \times$ “strategies".

The percentage contribution for a document with abstract "The study of cooperation in humans" has a $c_{A}=0.039+0.028+$ $0.026=0.093$ and $c_{B}=0.020+0.0+0.0=0.020$. The topic to which a document is assigned to is based on the highest percentage contribution denoted as $c^{*}$. For the given example the dominant topic is Topic A $c^{*}=c_{A}$.

$\mathrm{LAD}$ requires that the number of topics is specified in advance before running the algorithm. The appropriate number of topics can be chosen based on the coherence score (Röder et al., 2015) or the exclusivity score (Airoldi and Bischof, 2012). The coherence score measures the degree of semantic similarity between highly weighted words of a topic. There are cases for which a few topics can be dominated by very common words, and for that reason the exclusivity of words to topics has also been calculated. Figure 3 gives the topic coherence and the exclusivity of 18 models where the number of topics $n \in\{2,3, \ldots, 18\}$. The topic coherence for each model was calculated using the open source project (Rehurek and Sojka, 2010). The exclusivity measure was calculated with an altered version of (Rehurek and Sojka, 2010) which has been archived at (Glynatsi, 2020).

From Fig. 3 it can be seen that the number of topics with the highest coherence score are $n=6$ (coherence score of 0.418 ) and $n=12$ (coherence score of 0.417 ). Figure 3 also shows that the exclusivity of the highly weighted words of the topics is decreasing as the number of topics increases. A number of topics $n=5$ has a better exclusivity value than the model of $n=6$, and its coherence score is 0.406 (which is closed to 0.418). For that reason $n=5$ is chosen to carry out the analysis of this work.

For $n=5$ the articles are clustered and assigned to their dominant topic, based on the highest percentage contribution. The keywords associated with a topic, the most representative article of the topic (based on the percentage contribution) and its academic reference are given by Table 2 . The topics are labelled as A, B, C, D and E, and more specifically:

- Based on the keywords associated with Topic A, and the most representative article, Topic A appears to be about human subject research. Several publications assigned to the topic study the PD by setting experiments and having human participants simulate the game instead of computer simulations. These articles include (Matsumoto et al., 2016) which showed that prosocial behavior increased with the age of the participants, (Li et al., 2014) which studied the difference in cooperation between high-functioning autistic and typically developing children, (Molina et al., 2013) explored the gender effect in highschool students and (Bell et al., 2017) explored the effect of facial expressions of individuals.

- Though it is not immediate from the keywords associated with Topic B, investigating the papers assigned to the topic indicate that it is focused on biological studies. Papers assigned to the topic include papers which apply the PD to genetics (Sistrom et al., 2015), to the study of tumours (Sartakhti et al., 2017) and viruses (Turner and Chao, 1999). Other works include how phenotype affinity can affect the emergence of cooperation (Wu et al., 2019) and modeling bacterial communities as a spatial structured social dilemma.

- Based on the keywords and the most representative article Topic C appears to include publications on PD strategies. Publications in the topic include the introduction of new strategies (Stewart and Plotkin, 2013), the search of optimality in strategies (Banerjee and Sen, 2007) and the training of strategies (Ishibuchi et al., 2011) with different representation methods. Moreover, publications that study the evolutionary stability of strategies (Adami and Hintze, 2013) and introduced methods of differentiating between them (Ashlock and Kim, 2008) are also assigned to C.

- The keywords associated with Topic D clearly show that the topic is focused on evolutionary dynamics on networks. Publications include (Ichinose et al., 2013) which explored the robustness of cooperation on networks, (Wang et al., 2012) which studied the effect of a strategy's neighbourhood on the emergence of cooperation and (Chen et al., 2016) which explored the fixation probabilities of any two strategies is spatial structures.

- The publication assigned to Topic $\mathrm{E}$ are on modeling problems as a PD game. Though Topic $\mathrm{B}$ is also concerned with problems being formulated as a PD, it includes only biological problems. In comparison, the problems in Topic $\mathrm{E}$ include decision making in operational research (Ormerod, 2010), information sharing among members in a virtual team (Feng and Liu, 2008), the measurement of influence in articles based on citations (Hutchins et al., 2016) and the price spikes in electric power markets (Guan, 2002), and not on biological studies.

Note that the whilst for the choice of 5 topics the actual clustering is not subjective (the algorithm is determining the output) the interpretation above is.

Figure 4 gives the number of articles per topic over time. The topics appear to have had a similar trend over the years, with topics B and D having a later start. Following the introduction of a topic the publications in that topic have been increasing, and
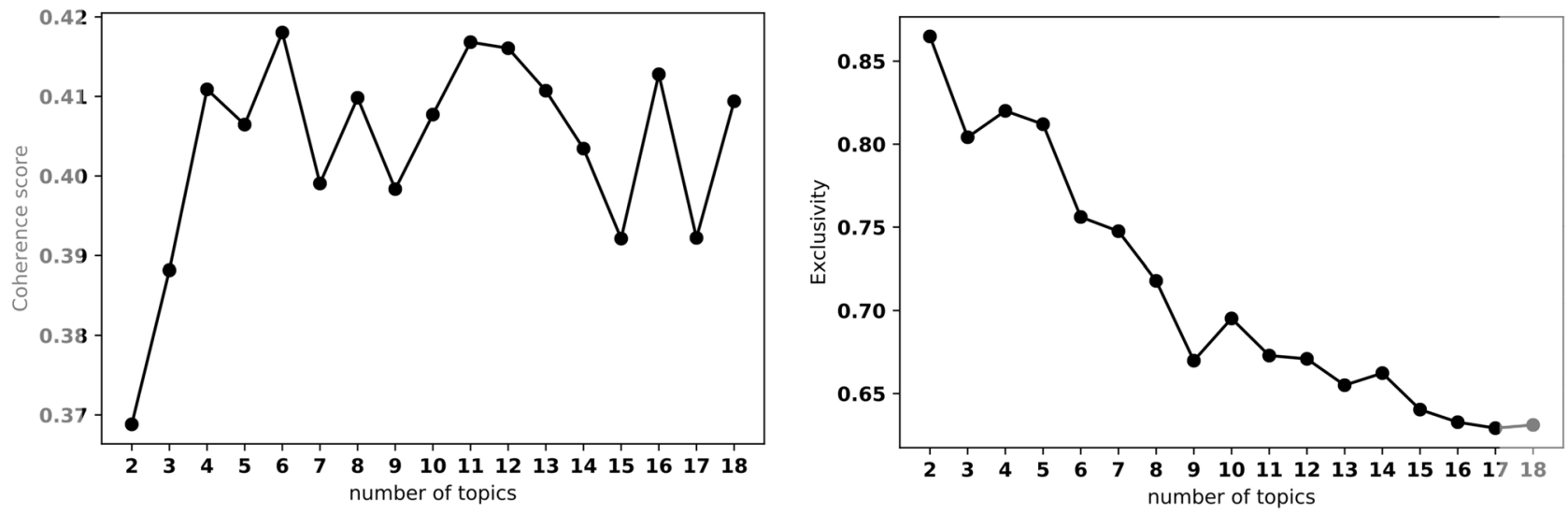

Fig. 3 Coherence and exclusivity for LDA models over the number of topics. The coherence score and the exclusivity of 18 models where the number of topics $n \in\{2,3, \ldots, 18\}$. 


\begin{tabular}{|c|c|c|c|c|c|}
\hline Dominant topic & Topic keywords & Most representative article title & Reference & \# Documents & $\%$ Documents \\
\hline $\bar{A}$ & $\begin{array}{l}\text { Social, behavior, human, study, } \\
\text { experiment, cooperative, cooperation, } \\
\text { suggest, find, behaviour }\end{array}$ & $\begin{array}{l}\text { Facing aggression: cues differ for female } \\
\text { versus male faces }\end{array}$ & $\begin{array}{l}\text { (Geniole et al., } \\
\text { 2012) }\end{array}$ & 496.0 & 0.2008 \\
\hline B & $\begin{array}{l}\text { Individual, group, good, show, high, } \\
\text { increase, punishment, cost, result, } \\
\text { benefit }\end{array}$ & $\begin{array}{l}\text { Genomic and gene-expression } \\
\text { comparisons among phage-resistant } \\
\text { type-IV Pilus mutants of Pseudomonas } \\
\text { syringae pathovar phaseolicola }\end{array}$ & $\begin{array}{l}\text { (Sistrom et al., } \\
\text { 2015) }\end{array}$ & 309.0 & 0.1251 \\
\hline C & $\begin{array}{l}\text { Game, strategy, player, agent, } \\
\text { dilemma, play, payoff, state, prisoner, } \\
\text { equilibrium }\end{array}$ & $\begin{array}{l}\text { Fingerprinting: visualization and } \\
\text { automatic analysis of prisoner's dilemma } \\
\text { strategies }\end{array}$ & $\begin{array}{l}\text { (Ashlock and } \\
\text { Kim, 2008) }\end{array}$ & 561.0 & 0.2271 \\
\hline D & $\begin{array}{l}\text { Cooperation, network, population, } \\
\text { evolutionary, evolution, interaction, } \\
\text { dynamic, structure, cooperator, study }\end{array}$ & $\begin{array}{l}\text { Influence of initial distributions on robust } \\
\text { cooperation in evolutionary prisoner's } \\
\text { dilemma }\end{array}$ & $\begin{array}{l}\text { (Chen et al., } \\
\text { 2007) }\end{array}$ & 556.0 & 0.2251 \\
\hline
\end{tabular}
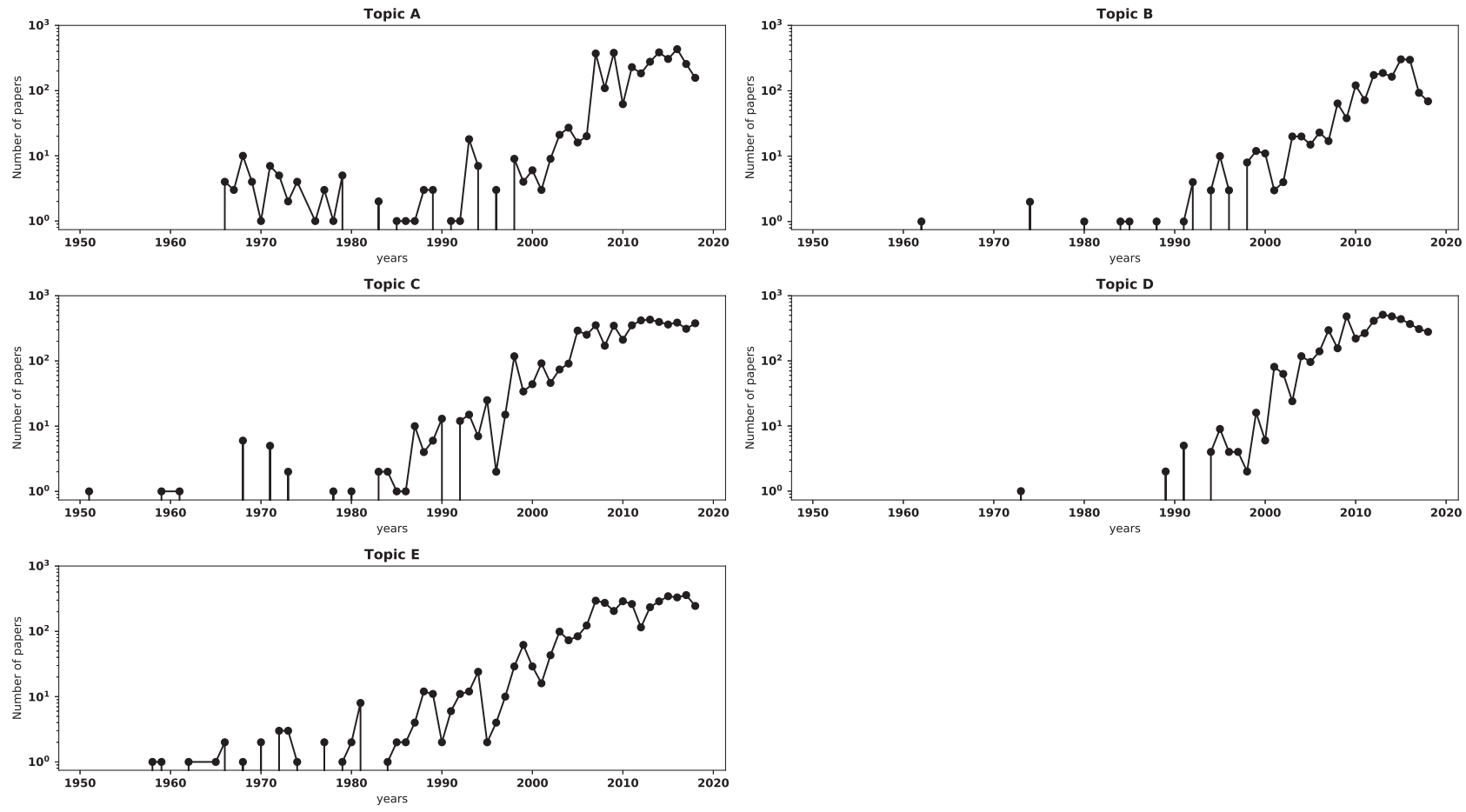

Fig. 4 Number of articles per topic over the years. The number of articles for Topics $A$ to $E$ on a log scale.

there is no decreasing trend in any of the topics. All the topics have been publishing for years and they still attract the interest of academics. Thus, there does not seem to be any given topic more or less in fashion.

To gain a better understanding regarding the change in the topics over the years, LDA is applied to the cumulative data set over 8 time periods. These periods are 1951-1965, 1951-1973, 1951-1980, 1951-1988, 1951-1995, 1951-2003, 1951-2010, 1951-2018. The number of topics for each cumulative subset is chosen based only on the topic coherence, and the exclusivity is not taken into account. As a result, the period 1951-2018 has been assigned $n=6$ which had the highest coherence value instead of 5 . The chosen models for each period including the number of topics, their keywords and number of articles assigned to them are given by Table S.1 (found in the Supplementary Information).
But how well do the five topics which were presented earlier fit the publications over time? This is answered by comparing the performance of three LDA models over the cumulative periods' publications. The three models are LDA models for the entire data set for $n$ equal to 5, 6 and the models of Table S.1 for each time period. Thus, for the period 1951-1980 the three model that are being compared are for $n$ equal to 5, 6, and 13 .

For each model the $c^{*}$ is estimated for each document in the cumulative data sets. The performance of the models are then compared based on:

$$
\overline{c^{*}} \times n
$$

where $\bar{c}^{*}$ is the median highest percentage contribution and $n$ is the number of topics of a given period. A model with more topics will have more difficulty to assign papers. Thus, Eq. (1) is a measure of confidence in assigning a given paper to its topic 

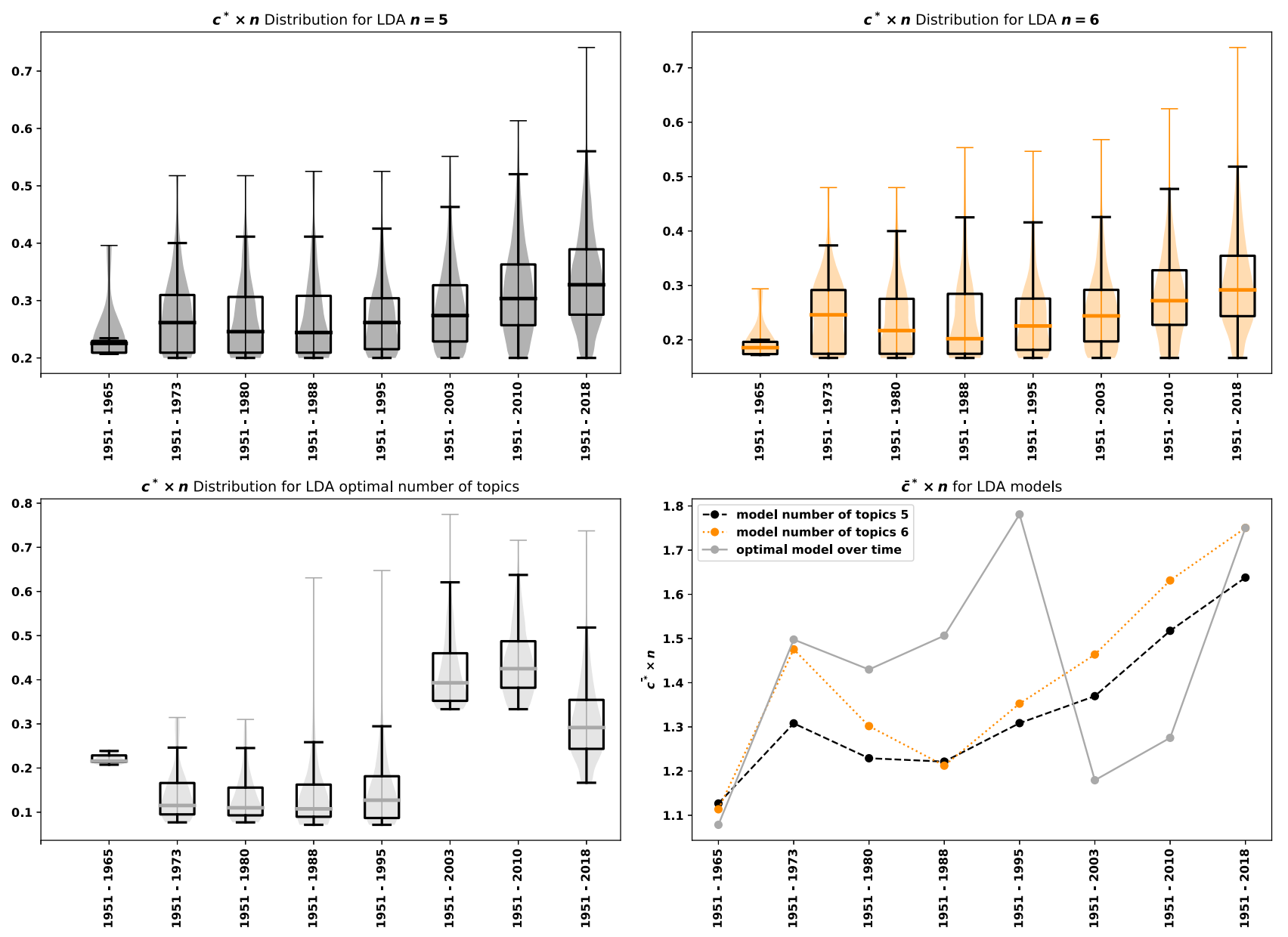

Fig. 5 Maximum percentage contributions over the time periods. The maximum percentage contributions $\left(c^{\star}\right)$ over the time periods for the LDA models for the entire data set for $n$ equal to 5, 6 and for the models of Table S.1.

weighted by the number of topics. The performances are given by Fig. 5.

The five topics of the PD presented in this manuscript appear to always be less good at fitting the publications compared to the six topics of LDA $n=6$. Moreover, these are less good than the models of periods 1951-1965 to 1951-1995. The difference in the performance values, Eq. (1), however is small. The relevances of the five topics has been increasing over time, and though, the topics did not always fit the majority of published work, there were still papers being published on those topics.

In the following section the collaborative behaviour of authors in the field, and within the field's topics as were presented in this section, are explored using a network theoretic approach.

Analysis of co-authorship network. The relationship between the authors within a field is modeled as a graph $G=\left(V_{G}, E_{G}\right)$ where $V_{G}$ is the set of nodes and $E_{G}$ is the set of edges. The set $V_{G}$ represents the authors and an edge connects two authors if and only if those authors have written together. This co-authorship network is constructed using the main data set (Glynatsi, 2019c) and the open source package (Hagberg et al., 2008). The PD network is denoted as $G$ where the number of unique authors $\mid V$ $(G) \mid$ is 4226 and $|E(G)|$ is 7642 .

The collaborativeness of the authors is analysed using measures such as, number of isolated nodes, number of connected components, clustering coefficient, number of communities, modularity and average degree. These measures show the number of connections authors can have and how strongly connected these people are. The number of isolated nodes is the number of nodes that are not connected to another node, thus the number of authors that have published alone. The average degree denotes the average number of neighbours for each nodes, i.e. the average number of collaborations between the authors. A connected component is a maximal set of nodes such that each pair of nodes is connected by a path (Easley et al., 2010). The number of connected components, as well as the size of the largest connected component in the network are reported. The size of the largest connected component represents the scale of the central cluster of the entire network. Clustering coefficient and modularity are also calculated. The clustering coefficient, defined as 3 times the number of triangles on the graph divided by the number of connected triples of nodes, is a local measure of the degree to which nodes in a graph tend to cluster together in a clique (Easley et al., 2010). It shows to which extent the collaborators of an author also write together. In comparison, modularity is a global measure designed to measure the strength of division of a network into communities. The number of communities is reported using the Clauset-Newman-Moore method (Clauset et al., 2004). Also the modularity index based on the Louvain method (Chen et al., 2007) is calculated using (Aynaud, 2020). The value of the modularity index can vary between $[-1,1]$, a high value of modularity corresponds to a structure where there are dense connections between the nodes within communities but sparse connections between nodes in different communities. That means that there are many sub communities of authors that write together but not across communities. Two centrality measures are also reported. These are: 
Table 3 Network metrics for G, $\bar{G}$, Auction games and Price of Anarchy.

\begin{tabular}{|c|c|c|c|c|c|c|c|c|c|c|}
\hline & \# Nodes & \# Edges & \# Isolated nodes & $\%$ Isolated nodes & $\begin{array}{l}\text { \# Connected } \\
\text { components }\end{array}$ & $\begin{array}{l}\text { Size of largest } \\
\text { component }\end{array}$ & Av. degree & \# Communities & Modularity & Clustering coeff \\
\hline$G$ & 4221 & 7642 & 338 & 8.0 & 1157 & 796 & 3.621 & 1177 & 0.965264 & 0.666 \\
\hline $\bar{G}$ & 796 & 2214 & 0 & 0.0 & 1 & 796 & 5.563 & 29 & 0.840138 & 0.773 \\
\hline Auction games & 5362 & 7861 & 453 & 8.4 & 1469 & 1348 & 2.932 & 1493 & 0.957238 & 0.599 \\
\hline Price of anarchy & 1315 & 1952 & 165 & 12.5 & 406 & 221 & 2.969 & 414 & 0.964498 & 0.626 \\
\hline
\end{tabular}

Table 4 Network metrics for topic networks.

\begin{tabular}{|c|c|c|c|c|c|c|c|c|c|c|}
\hline & \# Nodes & \# Edges & \# Isolated nodes & $\%$ Isolated nodes & $\begin{array}{l}\text { \# Connected } \\
\text { components }\end{array}$ & $\begin{array}{l}\text { Size of } \\
\text { largest } \\
\text { component }\end{array}$ & Av. degree & \# Communities & Modularity & Clustering coeff \\
\hline Topic A & 1193 & 2137 & 84 & 7.0 & 333 & 56 & 3.583 & 334 & 0.983 & 0.715 \\
\hline Topic B & 727 & 1382 & 45 & 6.2 & 189 & 80 & 3.802 & 190 & 0.950 & 0.739 \\
\hline Topic C & 931 & 1141 & 72 & 7.7 & 312 & 29 & 2.451 & 312 & 0.981 & 0.615 \\
\hline Topic D & 891 & 1509 & 28 & 3.1 & 185 & 312 & 3.387 & 193 & 0.917 & 0.692 \\
\hline Topic E & 1152 & 1964 & 166 & 14.4 & 461 & 31 & 3.410 & 461 & 0.926 & 0.602 \\
\hline
\end{tabular}

1. Closeness centrality, where a node is seen as centrally involved in the network if it requires only few intermediaries to contact others and thus is structurally relatively independent.

2. Betweenness centrality, where the determination of an author's centrality is based on the quotient of the number of all shortest paths between nodes in the network that include the node in question and the number of all shortest paths in the network. In betweenness centrality the position of the node matters.

There are a total of 1157 connected components in $G$ and the largest component has a size of 796 nodes. The largest connected component is going to be refereed to as the main cluster of the network and is denoted as $\bar{G}$. A metrics summary of both networks is given by Table 3 . Based on Table 3 an author in $G$ has on average 4 collaborators and a $67 \%$ probability of collaborating with a collaborator's co-author. An author of $\bar{G}$ on average is $10 \%$ more likely to write with a collaborator's coauthor and on average has 2 more collaborators. Moreover, there are only $8.0 \%$ of authors in the PD that have no connection to any other author.

How does this compare to other fields? Two more data sets for the topics "Price of Anarchy" and "Auction Games" have been collected in order to compare the collaborative behaviour of the PD to other game theoretic fields. A total of 3444 publications have been collected for Auction games and 748 for Price of Anarchy. Price of Anarchy is relatively a new field, with the first publication on the topic being (Koutsoupias and Papadimitriou, 1999) in 1999. This explains the small number of articles that have been retrieved. Both data sets have been archived and are available in (Glynatsi, 2019a, b). The networks for both data sets have been generated in the same way as $G$, and a summary of the networks' metrics is also given by Table 3 .

The average degrees for the Price of Anarchy and for Auction games are lower than the PD's, and so are their respective clustering coefficients. Moreover, both the Price of Anarchy and Auction games have a larger number of isolated authors. These results seem to indicate that the PD is a relatively collaborative field, compared to other game theoretic fields. However, both $G$ and $\bar{G}$ have a high modularity (larger than 0.84 ) and a large number of communities (967 and 25, respectively). A high modularity implies that authors create their own publishing communities but not many publications from authors from different communities occur. Thus, author tends to collaborate with authors in their communities but not many efforts are made to create new connections to other communities and spread the knowledge of the field across academic teams. The fields of both Price of Anarchy and Auction games also have high modularity, and that could indicate that is in fact how academic publications are.

The evolution of the networks was also explored over time by constructing the network cumulatively over 51 periods. Except from the first period 1951-1966 the rest of the periods have a yearly interval (data for the years 1975 and 1982 were not retrieved by the collection data process). The metrics of each sub network are given in the Supplementary Information. The results, similarly to the results of (Liu and Xia, 2015), confirm that the networks grow over time and that the networks always had a high modularity. Since the first publications authors tend to write with people from their communities, and that is not an effect of a specific time period.

The networks corresponding to the topics of Section "Research topics in the Prisoner's Dilemma research" have also been generated similarly to $G$. Note that authors with publications in more than one topic exist, and these authors are included in all the corresponding networks. A metrics' summary for all five topic networks is given by Table 4 .

Topics A and B have the highest average degree and clustering coefficient. Moreover, both topics have a small number of isolated nodes. Compared to that Topic $\mathrm{C}$ has a smallest average degree and Topic E has the highest number of isolated authors. These indicate that the topics "human subject research" and "biological studies" tend to be more collaborative than the topic of "strategies", and authors in these are more likely to have at least one collaborator compared to the topic of "modeling problems as a PD".

Topic "Evolutionary dynamics on networks" also appears to be a collaborative topic. It is the topic with smallest number of isolated authors, and has an average degree of 3.4. In fact the network of the topic is a sub graph of $\bar{G}$, the main cluster of $G$. This is discussed in the next part of this analysis.

There are two centrality measures reported in this work, closeness and betweenness centrality. Closeness centrality is a measure of how easy it is for an author to reach others, and betweenness centrality is a measure of how many paths pass through a specific node. All centrality measures have values ranging from 0 to 1 . 
Table 510 most central authors based on betweenness and closeness centralities for $\boldsymbol{G}$ and $\overline{\boldsymbol{G}}$.

G

\begin{tabular}{|c|c|c|c|c|c|c|c|c|}
\hline & Name & Betweenness & Name & Closeness & Name & Betweenness & Name & Closeness \\
\hline 1 & Matjaz Perc & 0.013 & Matjaz Perc & 0.062 & Matjaz Perc & 0.373 & Matjaz Perc & 0.330 \\
\hline 2 & Zhen Wang & 0.010 & Long Wang & 0.057 & Zhen Wang & 0.279 & Long Wang & 0.301 \\
\hline 3 & Long Wang & 0.006 & Yamir Moreno & 0.056 & Long Wang & 0.170 & Yamir Moreno & 0.299 \\
\hline 5 & Angel Sanchez & 0.004 & Zhen Wang & 0.056 & Angel Sanchez & 0.114 & Zhen Wang & 0.296 \\
\hline 6 & Yamir Moreno & 0.004 & Arne Traulsen & 0.053 & Yamir Moreno & 0.110 & Arne Traulsen & 0.281 \\
\hline 7 & Arne Traulsen & 0.004 & Luo-Luo Jiang & 0.053 & Arne Traulsen & 0.107 & Luo-Luo Jiang & 0.280 \\
\hline 10 & Valerio Capraro & 0.003 & Xiaojie Chen & 0.052 & Valerio Capraro & 0.093 & Xiaojie Chen & 0.276 \\
\hline
\end{tabular}

For $G$ and $\bar{G}$ the most central authors based on closeness and betweenness centralities are given by Table 5 . The most central authors in $G$ and $\bar{G}$ are the same. This implies that the results on centrality heavily rely on the main cluster (as expected). Matjaz Perc is an author with 83 publications in the data set and the most central authors based on both centrality measures. The most central authors are fairly similar between the two measures. The author that appear to be central based on one measure and not the other are Martin Nowak, Franz Weissing, Jianye Hao, Angel Sanchez and Valerio Capraro which are central based on betweeness centrality, and the opposite is true for Attila Szolnoki, Luo-Luo Jiang Sandro Meloni, Cheng-Yi Xia, and Xiaojie Chen.

The centrality measures for the topic networks have also been estimated and are given in Tables 6 and 7. The centrality measure for the topics' networks are low except from the case of Topic D. From the list of names it is obvious that the most central authors of Topic D are part of $\bar{G}$, and that the network of evolutionary dynamics on networks is a sub network of $\bar{G}$.

This confirms the result that the most central authors of the coauthorship network are the authors of the main cluster of $G$. The fact that most authors of the main cluster are primarily publishing in evolutionary dynamics on networks indicates that publishing in this specific topic differs from the other topics covered in this manuscript. It could also indicate that authors publishing in evolutionary dynamics are more similar to other disciplines as they can collaborate with them more.

The distributions of both centrality measures for all the networks of this work are given in the Supplementary Information.

\section{Conclusion}

This manuscript has explored the research topics in the publications of the Iterated Prisoner's Dilemma, and moreover, the authors' collaborative behaviour and their centrality. This was achieved by applying network theoretic approaches and a LDA algorithm to a total of 2422 publications. The data collection and an initial analysis of the data set were covered in Section "Methodology". The analysis demonstrated that the PD is a field that continues to attract academic attention and publications.

In Section "Research topics in the Prisoner's Dilemma research" LDA was applied to the data set to identify topics on which researchers have been publishing. The five topics in the PD publications identified by the data set of this work are human subject research, biological studies, strategies, evolutionary dynamics on networks and modeling problems as a PD. These 5 topics nicely summarise PD research. They highlight the interdisciplinarity of the field; how it brings together applied modeling of real world situations (biological studies and modeling problems as a PD) and more theoretical notions such as evolutionary dynamics and optimality of strategies. A temporal analysis explored how relevant these topics have been over the course of time, and it revealed that even though they were not necessarily always the most discussed topics there were still being explored by researchers.

The collaborative behaviour of the field was explored in Section "Analysis of co-authorship network" investigated the co-authorship network. It was concluded that the field is a collaborative field, where authors are likely to write with a collaborator's co-authors and on average an author has 4 co-authors. The results were compared to the networks of two other game theoretic fields, and it was shown that the PD network is relatively more collaborative. The authors however, tend to collaborate with authors from one community, but not many authors are involved in multiple communities. This might be an effect of academic research, and it might not be true just for the field of the PD.

Exploring the centrality of authors showed that the most central author of this manuscript is Matjaz Perc. More importantly, it was shown that most central authors of the network were the authors connected to the main cluster. Interestingly, it was uncovered that these authors were the most central due to their publication on a single topic alone. That was the topic of "evolutionary dynamics on networks". There appears to be more collaboration and more influence in the publications on evolutionary dynamics. The authors are most likely to gain from their position, and come across as the more important authors in the field. Though it is not clear as to why, attention should be paid to the collaborative behaviour of authors of "evolutionary dynamics on networks".

The study of the PD is the study of cooperation and investigating the cooperative behaviours of authors is what this work has aimed to achieve. Interesting areas of future work would include extending this analysis to more game theoretic sub fields, to evaluate whether the results remain the same. Moreover, the networks of this work were created by not taking into account the strength of ties. The strength of ties could be analysed to map multiple collaborations between two nodes. However, a preliminary assessment showed that the presented results do not change.

Both the software (Glynatsi, 2017) and the data (Glynatsi, 2019c) used in the manuscript have been archived and are available to be used by other researchers.

\section{Data availability}

The datasets generated and analysed during this study are available in (Glynatsi, 2019a, b, c). 

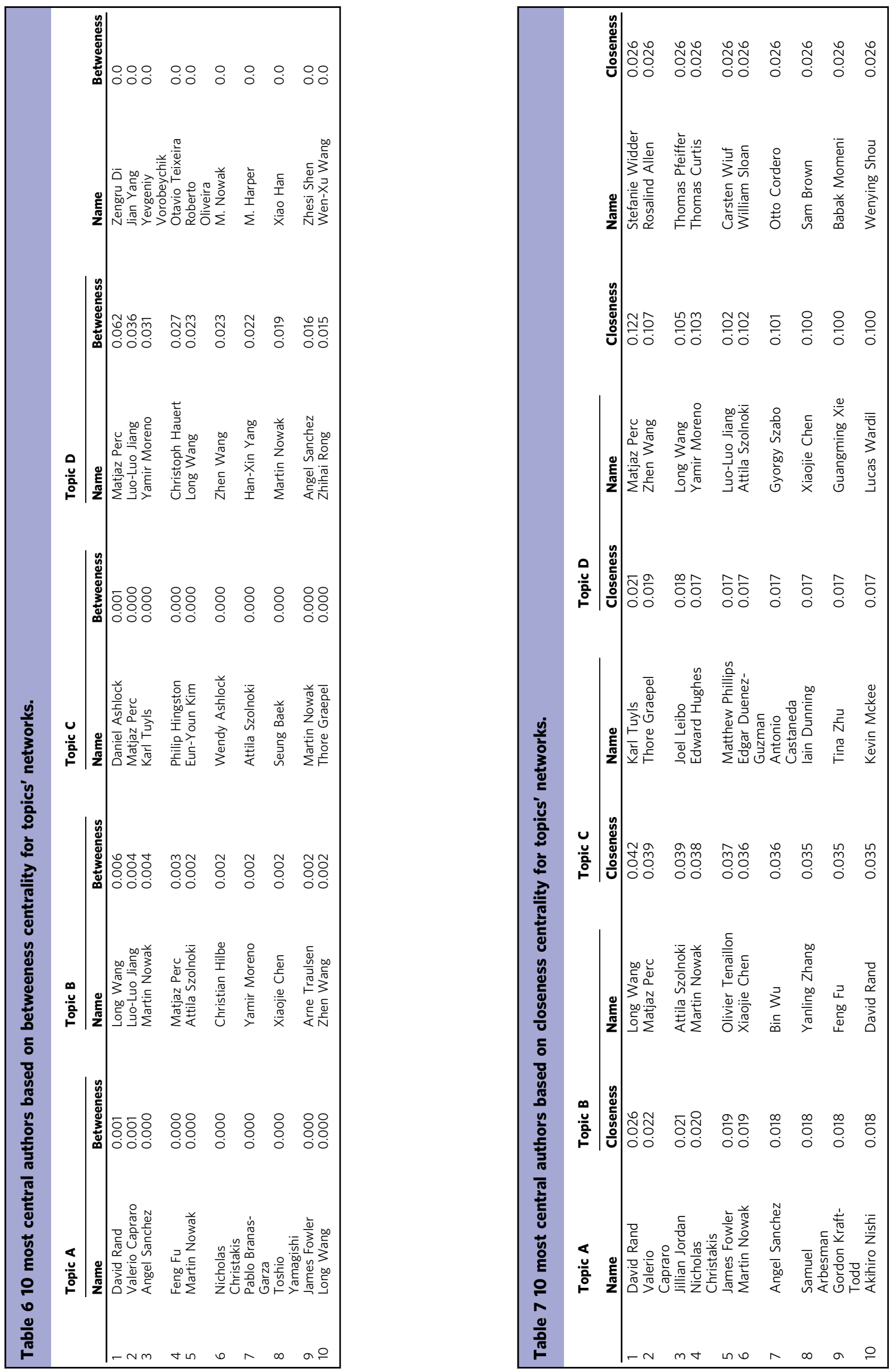
Received: 5 February 2020; Accepted: 11 January 2021;

Published online: 11 February 2021

\section{References}

Adami C, Hintze A (2013) Evolutionary instability of zero-determinant strategies demonstrates that winning is not everything. Nature communications 4:2193

Airoldi EM, Bischof JM (2012) A poisson convolution model for characterizing topical content with word frequency and exclusivity. Preprint at arXiv. 1206.4631

Ashlock D, Kim EY (2008) Fingerprinting: visualization and automatic analysis of prisoner's dilemma strategies. IEEE Trans Evolut Comput 12:647-659

Axelrod R (1980a) Effective choice in the prisoner's dilemma. J Conflict Resol 24:3-25

Axelrod R (1980b) More effective choice in the prisoner's dilemma. J Conflict Resol 24:379-403

Axelrod R, Hamilton WD (1981) The evolution of cooperation. Science 211:1390-1396

Axelrod R (1987) The evolution of strategies in the iterated prisoner's dilemma. In Davis, D. (ed.), Genetic Algorithms and Simulated Annealing pp. 1-16

Aynaud T (2020) python-louvain 0.14: Louvain algorithm for community detection. https://github.com/taynaud/python-louvain

Banerjee D, Sen S (2007) Reaching pareto-optimality in prisoner's dilemma using conditional joint action learning. Autonom Agents Multi-Agent Syst 15:91-108

Bell R, Mieth L, Buchner A (2017) Separating conditional and unconditional cooperation in a sequential prisoner's dilemma game. PloS one 12: e0187952

Bergmann T, Dale R (2018) A scientometric analysis of evolang: intersections and authorships. In The Evolution of Language: Proceedings of the 11th International Conference (EVOLANG11). New Orleans: Evolang Scientific Committee Vol. 22

Blei DM, Ng AY, Jordan MI (2003) Latent dirichlet allocation. J Machine Learn Res 3:993-1022

Blondel VD, Guillaume JL, Lambiotte R, Lefebvre E (2008) Fast unfolding of communities in large networks. J Statis Mechan 2008:P10008

Chen X, Fu F, Wang L (2008) Influence of different initial distributions on robust cooperation in scale-free networks: A comparative study. Physics Letters A 372:1161-1167

Chen YT, McAvoy A, Nowak MA (2016) Fixation probabilities for any configuration of two strategies on regular graphs. Sci Rep 6:39181

Clauset A, Newman MEJ, Moore C (2004) Finding community structure in very large networks. Phys Rev E 70(6):066111

Coelho LP, Peng T, Murphy RF (2010) Quantifying the distribution of probes between subcellular locations using unsupervised pattern unmixing. Bioinformatics 26:i7-i12

das NevesMachado R, Vargas-Quesada B, Leta J (2016) Intellectual structure in stem cell research: exploring brazilian scientific articles from 2001 to 2010 . Scientometrics 106:525-537

Easley D, Kleinberg J (2010) Networks, crowds, and markets. Cambridge: Cambridge University Press. 8th edn

Etzkowitz H (1992) Individual investigators and their research groups. Minerva 30:28-50

Feng X, Liu Y (2008) Trilateral game analysis on information sharing among members in a virtual team. In 2008 IEEE Symposium on Advanced Management of Information for Globalized Enterprises (AMIGE). IEEE, pp. 1-5

Flood MM (1958) Some experimental games. Manag Sci 5:5-26

Geniole SN, Keyes AE, Mondloch CJ, Carré JM, McCormick CM (2012) Facing aggression: cues differ for female versus male faces. PLoS ONE 7:e30366

Glynatsi NE (2017) Nikoleta-v3/arcas: Arcas v 0.0.4. https://doi.org/10.5281/ zenodo. 1127684

Glynatsi NE (2019a) Articles' meta data on the price of anarchy. https://doi.org/ 10.5281/zenodo. 3406542

Glynatsi NE (2019b) Articles' meta data on auction games. https://doi.org/10.5281/ zenodo. 3406544

Glynatsi NE (2019c) Articles' meta data on the prisoner's dilemma

Glynatsi NE (2020) Nikoleta-v3/gensim 1.0.0. https://doi.org/10.5281/ zenodo. 4290015

Grimmer J, Stewart BM (2013) Text as data: the promise and pitfalls of automatic content analysis methods for political texts. Polit Anal 21:267-297

Guan X (2002) Gaming and price spikes in electric power markets and possible remedies. In: Proceedings. International Conference on Power System Technology. Vol. 1, IEEE, pp. 188

Hagberg AA, Schult DA, Swart PJ (2008) Exploring network structure, dynamics, and function using NetworkX. In Proceedings of the 7th Python in Science Conference (SciPy) Pasadena, pp. 11-15

Hunter JD (2007) Matplotlib: a 2D graphics environment. Comput Sci Eng 9:90-95
Hutchins BI, Yuan X, Anderson JM, Santangelo GM (2016) Relative citation ratio ( $\mathrm{rcr}$ ): a new metric that uses citation rates to measure influence at the article level. PLoS Biol 14:e1002541

Ichinose G, Tenguishi Y, Tanizawa T (2013) Robustness of cooperation on scalefree networks under continuous topological change. Phys Rev E 88:052808

IEEE (1963) IEEE Xplore Digital Library. http://ieeexplore.ieee.org/Xplore/home.jsp.

Inglis M, Foster C (2018) Five decades of mathematics education research. J Res Math Educ 49:462-500

Ishibuchi H, Ohyanagi H, Nojima Y (2011) Evolution of strategies with different representation schemes in a spatial iterated prisoner's dilemma game. IEEE Trans Comput Intell AI Games 3:67-82

Jones E, Oliphant T, Peterson P (2001) SciPy: Open source scientific tools for Python. http://www.scipy.org/.

Koutsoupias E, Papadimitriou C (1999) Worst-case equilibria. In: Proceedings of the 16th Annual Conference on Theoretical Aspects of Computer Science, STACS'99. Springer-Verlag Berlin, Heidelberg, pp. 404-413

Kyvik S, Reymert I (2017) Research collaboration in groups and networks: differences across academic fields. Scientometrics 113:951-967

Li J, Zhu L, Gummerum M (2014) The relationship between moral judgment and cooperation in children with high-functioning autism. Sci Rep 4:4314

Li W, Aste T, Caccioli F, Livan G (2019) Early coauthorship with top scientists predicts success in academic careers. Nat Commun 10:2041-1723

Liu P, Xia H (2015) Structure and evolution of co-authorship network in an interdisciplinary research field. Scientometrics 103:101-134

Mallapaty S (2018) Paper authorship goes hyper. https://www.natureindex.com/ news-blog/paper-authorship-goes-hyper.

Mannheim Media (1950) Springer Publishing. http://www.springer.com/.

Matsumoto Y, Yamagishi T, Li Y, Kiyonari T (2016) Prosocial behavior increases with age across five economic games. PLoS ONE 11:e0158671

McKiernan G (2000) arxiv. org: the los alamos national laboratory e-print server. Int J Grey Literat 1:127-138

Molina JA et al. (2013) Gender differences in cooperation: experimental evidence on high school students. PLoS ONE 8:e83700

Nature Publishing Group (1869) Nature. https://www.nature.com/.

Nurseitov N, Paulson M, Reynolds R, Izurieta C (2009) Comparison of json and xml data interchange formats: a case study. Caine 2009:157-162

Ohtsuki H, Hauert C, Lieberman E, Nowak MA (2006) A simple rule for the evolution of cooperation on graphs and social networks. Nature 441:502

Ormerod RJ (2010) Or as rational choice: a decision and game theory perspective. J Operat Res Soc 61:1761-1776

PLOS (2000) PLOS public library of science. https://www.plos.org/.

Pritchard A et al. (1969) Statistical bibliography or bibliometrics. J Document 25:348-349

Raina D, Gupta BM (1998) Four aspects of the institutionalization of physics research in india (1990-1950): Substantiating the claims of histortical sociology through bibliometrics. Scientometrics $42: 17-40$

Rehurek R, Sojka P (2010) Software framework for topic modelling with large corpora. In: Proceedings of the LREC 2010 Workshop on New Challenges for NLP Frameworks. Citeseer

Riolo RL, Cohen MD, Axelrod R (2001) Evolution of cooperation without reciprocity. Nature 414:441

Röder M, Both A, Hinneburg A (2015) Exploring the space of topic coherence measures. In: Proceedings of the eighth ACM international conference on Web search and data mining. ACM, pp. 399-408

Sartakhti JS, Manshaei MH, Basanta D, Sadeghi M (2017) Evolutionary emergence of angiogenesis in avascular tumors using a spatial public goods game. PLoS ONE 12:e0175063

Sekara V et al. (2018) The chaperone effect in scientific publishing. Proc Natl Acad Sci USA 115:12603-12607

Sistrom M et al. (2015) Genomic and gene-expression comparisons among phageresistant type-iv pilus mutants of pseudomonas syringae pathovar phaseolicola. PLoS ONE 10:e0144514

Stewart AJ, Plotkin JB (2012) Extortion and cooperation in the prisoner's dilemma. Proc Natl Acad Sci USA 109:10134-10135

Stewart AJ, Plotkin JB (2013) From extortion to generosity, evolution in the iterated prisoner's dilemma. Proc Nattl Acad Sci USA 110:15348-15353

Sugimoto CR, Li D, Russell TG, Finlay SC, Ding Y (2011) The shifting sands of disciplinary development: analyzing north american library and information science dissertations using latent dirichlet allocation. J Am Soc Inform Sci Technology 62:185-204

Turner PE, Chao L (1999) Prisoneras dilemma in an rna virus. Nature 398:441

van Noorden R (2017) The science that's never been cited. https://www.nature. com/articles/d41586-017-08404-0.

Walt S, Colbert SC, Varoquaux G (2011) The NumPy array: a structure for efficient numerical computation. Comput Sci Eng 13:22-30

Wang J, Xia CY, W YL, Ding S, Sun JQ (2012) Spatial prisoner's dilemma games with increasing size of the interaction neighborhood on regular lattices. Chinese Sci Bullet 57:724-728 
Wang X, Ma X, Grimson WEL (2008) Unsupervised activity perception in crowded and complicated scenes using hierarchical bayesian models. IEEE Trans Pattern Anal Machine Intell 31:539-555

Wu T, Fu F, Wang L (2019) Phenotype affinity mediated interactions can facilitate the evolution of cooperation. J Theor Biol 462:361-369

Youngblood M, Lahti D (2018) A bibliometric analysis of the interdisciplinary field of cultural evolution. Pal Commun 4:120

\section{Acknowledgements}

The authors would like to thank the anonymous reviewers for their comments which helped improve the paper.

\section{Funding}

Open Access funding enabled and organized by Projekt DEAL.

\section{Competing interests}

The authors declare no competing interests.

\section{Additional information}

Supplementary information The online version contains supplementary material available at https://doi.org/10.1057/s41599-021-00718-9.
Correspondence and requests for materials should be addressed to N.E.G.

Reprints and permission information is available at http://www.nature.com/reprints

Publisher's note Springer Nature remains neutral with regard to jurisdictional claims in published maps and institutional affiliations.

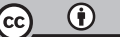

Open Access This article is licensed under a Creative Commons Attribution 4.0 International License, which permits use, sharing, adaptation, distribution and reproduction in any medium or format, as long as you give appropriate credit to the original author(s) and the source, provide a link to the Creative Commons license, and indicate if changes were made. The images or other third party material in this article are included in the article's Creative Commons license, unless indicated otherwise in a credit line to the material. If material is not included in the article's Creative Commons license and your intended use is not permitted by statutory regulation or exceeds the permitted use, you will need to obtain permission directly from the copyright holder. To view a copy of this license, visit http://creativecommons.org/ licenses/by/4.0/.

(C) The Author(s) 2021 